Ann. Génét. Sél. anim., I97x, 3 (4), 479-485.

\title{
EFFET D'UNE SÉLECTION AVEC TRONCATURE SUR DES GENES « IMPORTANTS » OU NON : QUELQUES ILLUSTRATIONS NUMÉRIQUES BASÉES SUR DES RELATIONS APPROXIMATIVES DANS LA LOI NORMALE
}

\author{
P. MÉRAT \\ Laboratoire de Génétique factorielle, \\ Centre national de Recherches zootechniques, I. N.R. A., \\ 78 - Jouy-en-Josas
}

\section{RÉSUMÉ}

\begin{abstract}
Faisant suite à un article antérieur (MÉRAT, I969), nous avons voulu préciser numériquement les cas où la réponse d'un gène à effet important (" majeur ") vis-à-vis d'une sélection avec troncature sur un caractère quantitatif diffère réellement de celle d'un gène à petit effet (" polygène ") : fréquence d'équilibre dépendant de l'intensité de sélection dans le cas d'un gène " majeur " avec " overdominance "; interaction mutuelle de gènes " majeurs" entre eux et avec les "polygènes " résiduels pour l'évolution de leurs fréquences.

En utilisant des relations linéaires approchées dans certains intervalles pour la loi normale, on précise que ces différences théoriquement prévisibles suivant l'importance de l'effet individuel d'un gène peuvent en première approximation grossière être négligées lorsque les différences entre génotypes au locus " majeur " considéré ne sont pas extrêmes (ne dépassant pas I à I,5 écart-type résiduel) et lorsque, de plus, le pourcentage global d'individus gardés comme reproducteurs est au moins égal à 20 ou $30 \mathrm{p}$. I0o, sans excéder 80 à $90 \mathrm{p}$. Ioo. En dehors de ces conditions, la prévision concernant la fréquence d'un gène " majeur " ne peut plus être assimilée à celle concernant un gène d'effet petit.
\end{abstract}

\section{INTRODUCTION}

Dans un article antérieur (MÉRAT, I969), nous avons souligné certaines différences de comportement entre gènes d'effet " important " ou non vis-à-vis d'une sélection avec troncature sur un caractère quantitatif. En particulier, dans les cas envisagés d'équilibre des fréquences alléliques, cet équilibre ne dépend de l'intensité 
de sélection que pour les gènes du premier type. Nous appellerons par convention " majeurs " ces loci pour lesquels les différences moyennes entre génotypes ne sont pas petites devant l'écart-type " résiduel ", le terme de " polygènes » désignant les autres loci.

D'autre part, ces gènes " majeurs ", lors d'une sélection avec pourcentage global constant d'individus gardés comme reproducteurs, sont nécessairement en interaction mutuelle, ainsi qu'avec les " polygènes " résiduels, pour l'évolution de leurs fréquences. Réciproquement, l'intensité de la sélection exercée sur les gènes " résiduels " est modifiée par la présence d'une ségrégation pour un gène " majeur ».

Il est souhaitable de préciser davantage jusqu'à quel point et dans quelles conditions (pression de sélection, importance de l'effet moyen d'un gène relativement à la variance résiduelle...) ces différences établies théoriquement entre gènes à effet individuel appréciable et gènes à petit effet revêtent une importance pratique réelle.

\section{I. - FRÉQUENCE D'ÉQUILIBRE POUR UN LOCUS AVEC “ OVERDOMINANCE » : DÉPENDANCE OU NON VIS-A-VIS DU POURCENTAGE DE GARDÉS}

a) Pour mieux circonscrire ces conditions, nous nous aiderons de relations linéaires approximatives obtenues empiriquement dans la loi normale.

Dans une distribution normale réduite soumise à une sélection par troncature, soit $t$ l'abscisse du point de troncature, $v$ le pourcentage de gardés, $\bar{\imath}$ leur valeur moyenne ou "intensité de sélection »(FALCONER, I960).

Plusieurs auteurs ont fait état de relations algébriques approchées entre ces variables : $t$ en fonction de $v$ et de $\bar{l}$ (LATTER, I965), $\bar{l}$ en fonction de $v$ (SMith, I969); mais la relation la plus simple, linéaire, est proposée par TIKU (I967, I968) entre $i$ et $t$ dans des intervalles d'amplitude limitée.

On peut vérifier empiriquement l'existence d'une relation linéaire approximative non seulement dans ce dernier cas, mais entre les trois variables prises deux à deux, pour des intervalles assez larges si l'on ne cherche pas une précision extrême. - Entre $v$ et $t$, la relation, symétrique par rapport au point $(t=0, v=0,5)$ avoisine 1a linéarité dans l'intervalle $0,3-0,7$ pour $v$ (erreurs absolues maxima sur $v$ de 1'ordre de 0,005 ) ou même $0,2-0,8$ (erreurs absolues ne dépassant pas 0,0I). Dans ce dernier cas, on peut écrire : $v \simeq-0,37 t+0,50$.

- Entre $\bar{l}$ et $v$, on peut appliquer la relation :

$$
\bar{\imath} \simeq-\mathrm{I}, 49^{v}+\mathrm{I}, 54
$$

pour des valeurs non extrêmes de $v$. L'approximation est bonne dans l'intervalle $0,5-0,9$ pour $v$ (erreurs sur $\vec{l}$ ne dépassant pas la troisième décimale), plus grossière pour $v$ compris entre 0,3 ou même 0,2 et 0,9 .

- Entre $i$ et $t$, la relation proposée par TrkU (I968) ne conduit pas à des erreurs très grandes encore pour $v$ compris entre 0,2 et 0,8 ou 0,9 .

Pour une comparaison entre comportement des " gènes majeurs " et des " polygènes ", il n'est pas nécessaire d'être extrêmement exigeant sur la qualité des approxi- 
mations indiquées ci-dessus, dès lors que nous voulons simplement situer les cas où ce comportement diffère beaucoup ou peu. C'est en ce sens que nous pouvons encore nous servir d'approximations comportant une erreur absolue de l'ordre de o,or.

b) Utilisation de ces relations.

Nous avons indiqué (MÉRAT, I969) que, pour un pourcentage global fixe $v$ d'individus gardés pour la reproduction dans une grande population, la fréquence d'équilibre $p_{0}$ pour un gène "majeur " à deux allèles $A$ et $a$ présentant une supériorité de 1'hétérozygote pour le caractère quantitatif sélectionné vérifie les deux équations

$$
\begin{gathered}
p_{0}=\frac{v_{\mathrm{AA}}-v_{a a}}{2 v_{\mathrm{A} a}-v_{\mathrm{AA}}-v_{a a}} \\
v=p_{0} v_{\mathrm{AA}}+2 p_{0}\left(\mathrm{I}-p_{0}\right) v_{\mathrm{A} a}+\left(\mathrm{I}-p_{0}\right)^{2} v_{a a}
\end{gathered}
$$

en appelant $v_{\mathrm{AA}}$, etc., les pourcentages de gardés relatifs à chaque génotype au locus A. Cette fréquence dépend de l'intensité de sélection exercée, alors que, pour un gène dont l'effet individuel est petit en regard de l'écart-type résiduel supposé constant, $p_{0}$ est pratiquement indépendant de cette intensité.

Supposons que nous soyons dans l'intervalle convenable pour exprimer linéairement chacun des $v$ ci-dessus en fonction de l'abscisse du point de troncature dans dans la distribution correspondante.

Appelons $t$ l'écart-réduit du point de troncature pour les A $a$. Il lui correspondra $t+\mathrm{d}$ pour les $\mathrm{AA}$ et $t+\mathrm{d}^{\prime}$ pour les $a a$, d et $\mathrm{d}^{\prime}$ étant respectivement la différence de la valeur moyenne des $\mathrm{A} a$ avec celle des AA et celle des $a a$.

Notons par $v \simeq \alpha t+\beta$ la relation linéaire approximative utilisée. L'équation ( $\mathrm{I}$ ) deviendra

$$
p_{0} \simeq \frac{\alpha t+\beta-\left[\alpha\left(t+\mathrm{d}^{\prime}\right)+\beta\right]}{2(\alpha t+\beta)-[\alpha(t+\mathrm{d})+\beta]-\left[\alpha\left(t+\mathrm{d}^{\prime}\right)+\beta\right]}=\frac{\mathrm{d}^{\prime}}{\mathrm{d}+\mathrm{d}^{\prime}}
$$

On obtient ainsi, y compris pour un gène d'effet relativement important, c'està-dire pour des valeurs appréciables de $d$ et $d^{\prime}$, une fréquence d'équilibre à peu près constante, indépendante de l'intensité de sélection.

L'influence de cette dernière ne sera donc vraiment sensible que lorsque l'un ou l'autre des pourcentages de gardés relatifs aux divers génotypes sera trop extrême - grosso modo hors de l'intervalle 20 p. roo-80 p. Ioo - pour permettre 1'approximation linéaire employée. Ceci se produira, soit si l'intensité de sélection globale est très élevée ou très faible, soit si les différences moyennes entre génotypes sont trop importantes, exprimées en écart-type résiduel (supérieures à $I$ ou $I, 5$ pour fixer les idées).

Numériquement, ces considérations se vérifient dans le tableau I de notre article précédent (MÉRAT, I969). Dans quatre situations envisagéas pour les valeurs de $\mathrm{d}$ et $\mathrm{d}^{\prime}$, la fréquence d'équilibre $p_{0}=\frac{\mathrm{d}^{\prime}}{\mathrm{d}+\mathrm{d}^{\prime}}$ qui correspondrait au cas " polygénique " ( $\mathrm{d}$ et $\mathrm{d}$ ' petits, mais dans le même rapport) est respectivement $0,75^{\circ}$, $0,857,0,857$ et 0,667 . On vérifie qu'on ne s'en écarte de façon importante, pour $\mathrm{d}$ et $\mathrm{d}^{\prime}$ ayant des valeurs appréciables, que pour un pourcentage global de gardés faible, inférieur à 20-30 p. Ioo. Ce sera seulement dans ces cas que, comme nous l'avons signalé, la variance " additive " associée au locus en question restera sensi- 
blement différente de zéro à l'équilibre, et qu'une intensification de la sélection pourra diminuer la moyenne d'équilibre.

On montrerait, de même, que, pour un gène " majeur " influant sur la variance, l'intensité de sélection n'influe pas sur les fréquences d'équilibre possibles, de même que pour un gène à petit effet, si les $v$ peuvent s'exprimer linéairement en fonction des $t$.

D’une façon plus générale, on peut conjecturer que 1'interdépendance " obligée " de gènes " majeurs " non allèles dans leur réponse à la sélection (MÉRAr, I969) ne sera réellement à considérer que dans les conditions où les approximations linéaires ci-dessus ne peuvent s'appliquer.

On pourrait aboutir aux mêmes conclusions de façon indirecte, en considérant $\Delta_{p}$, variation de la fréquence de $\mathrm{A}$ d'une génération à la suivante. Pour un gène à effet important, $\Delta_{p}$ s'exprime en fonction de $p$ et des pourcentages de gardés $v_{\mathbf{A} A}$, etc. dans chaque génotype. Si l'on peut appliquer l'approximation linéaire $v \simeq a \bar{\imath}+b$, on en tire l'expression de $\Delta_{p}$ en fonction de $p$ et des intensités de sélection $\bar{l}_{\mathrm{AA}}$, etc., et vérifie que 1 'on retombe approximativement sur la formule utilisée dans le cas polygénique.

\section{II. - EFFE'T DE LA SÉLECTION SUR LES “ POLYGÈNES » COMPARÉE EN L'ABSENCE OU EN PRÉSENCE D'UNE SÉGRÉGATION POUR UN GÈNE MAJEUR}

Nous ferons cette comparaison à pourcentage de gardés $v$ égal.

A $v$ correspond, en l'absence de ségrégation pour un gène majeur, une intensité de sélection $\bar{l}$, écart entre moyenne des gardés et moyenne générale.

En présence d'une ségrégation pour un gène " majeur » à deux allèles $\mathrm{A}, a$ donnant trois phénotypes distincts, on a, pour un même $v$ global :

$$
v=p^{2} v_{\mathrm{AA}}+2 p(\mathrm{I}-p) v_{\mathrm{A} a}+(\mathrm{I}-p)^{2} v_{a a}
$$

en appelant $p$ la fréquence du gène, $v_{\mathrm{A} A}$, etc.. les pourcentages de gardés pour chaque génotype au locus $\mathrm{A}$.

Soit $\bar{\imath}^{\prime}$ l'intensité globale de sélection sur les "polygènes " dans cette situation. En appelant $\bar{l}_{\mathrm{AA}}$, etc. les intensités " partielles " de sélection sur ces polygènes dans les distributions des individus AA, etc.., $i^{\prime}$ sera défini par

$$
\bar{l}^{\prime}=\frac{p^{2} v_{\mathrm{AA}} \bar{l}_{\mathrm{AA}}+2 p(\mathrm{I}-p) v_{\mathrm{A} a} \bar{l}_{\mathrm{A} a}+(\mathrm{I}-p)^{2} v_{a a} \bar{l}_{a a}}{p^{2} v_{\mathrm{AA}}+2 p(\mathrm{I}-p) v_{\mathrm{A} a}+(\mathrm{I}-p)^{2} v_{a a}}
$$

L'utilisation de l'approximation linéaire des $v$ en fonction des $\bar{\imath}$ montre qu'en général $\bar{l}^{\prime}$ est différent de $\bar{l}$ (alors que dans les mêmes conditions concernant les intensités de sélection on vérifie que $\bar{\imath} \simeq \bar{l}^{\prime}$ si la sélection est faite à point de troncature fixe).

On vérifie sur des exemples numériques que la présence d'une ségrégation à un locus " majeur " peut diminuer de façon assez importante, dans certains cas, l'intensité de sélection sur les autres gènes. 
Ainsi, prenons l'exemple simple du maintien d'un polymorphisme au locus A avec supériorité moyenne de l'hétérozygote $\mathrm{A} a$ et égalité des moyennes des deux génotypes AA et $a a$. A l'équilibre, la population se compose en proportion égale de deux distributions, celle des hétérozygotes, celle des homozygotes des deux génotypes.

Le tableau suivant donne l'intensité de sélection comparée sur les " polygènes " dans la population en question et dans une population sans ségrégation pour $\mathrm{A}$ et de même variance résiduelle, lorsque la différence moyenne $d$ entre les hétérozygotes $\mathrm{A} a$ et les homozygotes $\mathrm{AA}$ ou $a a$ est égale à I ou à 2 écarts-types des distributions résiduelles.

\section{TABLEAU I}

Intensité de sélection comparée sur les "polygènes" en présence ou en l'absence d'un équilibre des fréquences $\left(p=\frac{\mathrm{I}}{2}\right)$

au locus "majeur" $A$

$d$ représente la différence moyenne entre génotypes au locus $\mathrm{A}$, exprimée en écart-type résiduel.

Les pourcentages globaux de gardés correspondent respectivement aux valeurs - 2, - I, o et $+\mathrm{r}, \mathrm{o}$ du point de troncature dans la distribution des Aa.

\begin{tabular}{|c|c|c|c|c|}
\hline \multirow{2}{*}{ Pour $d=1$} & \multicolumn{4}{|c|}{ Pourcentage global de gardés } \\
\hline & 90,9 & 67,0 & 33,0 & 8,6 \\
\hline $\begin{array}{c}\text { Intensité de sé- } \\
\text { lection sur les } \\
\text { "polygènes" }\end{array}\left\{\begin{array}{l}\text { phisme polymor- } \\
\text { sans polymor- } \\
\text { phisme pour A }\end{array}\right.$ & $\begin{array}{l}0,163 \\
0,180\end{array}$ & $\begin{array}{l}0,478 \\
0,540\end{array}$ & $\begin{array}{l}0,973 \\
1,098\end{array}$ & $\begin{array}{l}1,626 \\
1,814\end{array}$ \\
\hline \multirow{2}{*}{ Pour $d=2$} & \multicolumn{4}{|c|}{ Pourcentage global de gardés } \\
\hline & 73,8 & 50,0 & 26,2 & 8,0 \\
\hline $\begin{array}{c}\text { Intensité de sé- } \\
\text { lection sur les } \\
\text { "polygènes" }\end{array}\left\{\begin{array}{l}\text { phisme pour A } \\
\text { sans polymor- } \\
\text { phisme pour A }\end{array}\right.$ & $\begin{array}{l}0,306 \\
0,440\end{array}$ & $\begin{array}{l}0,485 \\
0,798\end{array}$ & $\begin{array}{l}0,866 \\
1,240\end{array}$ & $\begin{array}{l}1,5 / 4 \\
1,871\end{array}$ \\
\hline
\end{tabular}

On voit que la diminution d'intensité de la sélection " polygénique ", sensible mais encore limitée pour une différence d'un écart-type entre génotypes au locus " majeur ", peut être d'un tiers à presque moitié lorsque cette différence atteint deux écarts-types.

Ainsi, le maintien d'un équilibre des fréquences géniques pour un gène à effet important, qui correspond déjà par lui-même au maintien d'une variabilité génétique " non sélectionnable ", peut au surplus " freiner " de façon appréciable la sélection aux autres loci, diminuant encore d’autant la réponse possible à cette sélection. 


\section{CONCLUSION}

Concernant la dépendance de la fréquence d'équilibre vis-à-vis de l'intensité de sélection pour un gène d'effet important présentant une supériorité de l'hétérozygote dans une grande population en panmixie, la relation approximative utilisée montre que cette dépendance peut être pratiquement négligée lorsque les différences entre génotypes au locus considéré ne sont pas extrêmes (ne dépassant pas I ou I,5 écart-type résiduel) et lorsque de plus, le pourcentage global d'individus gardés comme reproducteurs a une valeur d'au moins 20 ou $30 \mathrm{p}$. roo, sans excéder 80 à 90 p. I00. En dehors de ces conditions, nos conclusions antérieures sur le comportement particulier des gènes " majeurs" peuvent avoir une portée pratique.

Le deuxième point illustré numériquement montre la mesure non négligeable dans laquelle l'intensité de sélection sur la variabilité génétique résiduelle peut être diminuée lorsqu'une ségrégation est maintenue pour un gène majeur.

Cela confirme dans des cas précis le rôle spécial pouvant être joué en sélection par la présence de gènes à effet important.

Reçu pour publication en aồt 1971.

\section{SUMMARY}

EFFECT OF A TRUNCATION SELECTION ON GENES WITH OR WITHOUT " LARGE " EFFECT : SOME NUMERICAI, EXAMPLES BASED ON APPROXIMATE RELATIONS IN THE NORMAI, DISTRIBUTION

Following a previous paper (MÉRAT, I969), we attempted to specify numerically the cases when the response of a large effect (" major") gene to truncation selection on a quantitative trait differs from that of a small effect gene ("polygene") : Equilibrium frequency depending on selection intensity in the case of a "major " gene with overdominance ; mutual interaction of "major genes" with each other and with the residual " polygenes " for the evolution of their frequencies.

Making use of approximate linear relationships in certain intervals for the normal distribution, it is specified that these theoretically anticipated differences depending on the magnitude of the individual effect of a gene may be neglected as a very rough approximation when differences between genotypes at the "major " locus considered are not too large (inferior to I-I, 5 residual standard deviation) and when the overall percentage of selected individuals is superior to 20 $30 \mathrm{p}$. I 00 without exceeding $80-90 \mathrm{p}$. Ioo. Outside this set of conditions, the expectation for the frequency of a " major " gene can no more be assimilated to that concerning a small effect gene.

\section{RÉFÉRENCES BIBLIOGRAPHIQUES}

Falconer D. S., 1960. Introduction to quantitative genetics. Oliver and Boyd, London.

LAtTER B. D. H., I965. The response to artificial selection due to autosomal genes of large effect. I. Changes in gene frequency at an additive locus. Aust. J. Biol. Sci., 18, 585-598.

MÉrat P., I969. Rôle de l'intensité d'une sélection artificielle en présence de gènes à effet individuel 

important ou non. I. Sélection individuelle avec troncature, modèle déterministe. Ann. Génét. Sélect.
anim., 1, 243-272.

Sмıтн C., r969. Optimum selection procedures in animal breeding. Anim. Prod., 11, 433-442. TIKU M. L., I967. Estimating the mean and standard deviation from a censored normal sample.
Biometrika, 54, I55-165.

TikU M. L., I968. Estimating the parameters of normal and logistic distributions from censored samples. Aust. J. Statist., 10, 64-74. 